\title{
ON THE SOLVABILITY OF A CLASS OF REACTION-DIFFUSION SYSTEMS
}

\author{
ABDELFATAH BOUZIANI AND ILHAM MOUNIR
}

Received 30 October 2005; Accepted 4 January 2006

We deal with a class of parabolic reaction-diffusion systems. We use an iterative process based on results obtained for a linearized problem, then we derive some a priori estimates to establish the existence, uniqueness, and continuous dependence of the weak solution for a class of quasilinear systems.

Copyright (c) 2006 A. Bouziani and I. Mounir. This is an open access article distributed under the Creative Commons Attribution License, which permits unrestricted use, distribution, and reproduction in any medium, provided the original work is properly cited.

\section{Introduction}

Reaction-diffusion systems of PDEs furnish valuable mathematical models for a great number of phenomena in engineering and biology. For instance, the following system describes the dynamics of a simple isothermal chemical reaction system [25]:

$$
\begin{gathered}
\frac{\partial u}{\partial t}-\frac{\partial^{2} u}{\partial x^{2}}=u v \\
\frac{\partial v}{\partial t}-\frac{\partial^{2} v}{\partial x^{2}}=u v-h v
\end{gathered}
$$

where $h$ is a positive parameter. Moreover, the next system is a model for the description of the patchy distributions of microscopic aquatic organisms known as plankton (see, [17]):

$$
\begin{aligned}
& \frac{\partial u}{\partial t}-\frac{\partial^{2} u}{\partial x^{2}}=f_{1}(u, v), \\
& \frac{\partial v}{\partial t}-\frac{\partial^{2} v}{\partial x^{2}}=f_{2}(u, v)
\end{aligned}
$$


2 Solvability of a class of reaction-diffusion systems

where

$$
f_{1}(u, v)=a\left(a_{1}+b_{1} u+u^{2}\right) u-u v, \quad f_{2}(u, v)=u v-\left(a_{2} v+b_{2} v^{2}\right)
$$

and $\left(a, a_{1}, a_{2}, b_{1}, b_{2}\right)$ are positive parameters. Likewise, if $u(x, t)$ represents the population density, $v(x, t)$ the concentration of the attractant, $F(u)$ and $H(u, v)$ describe the local kinetics of the population and the attractant respectively, $t$ is the time, and $x$ is the onedimensional spatial variable, then the system

$$
\begin{gathered}
\frac{\partial u}{\partial t}-d_{1} \frac{\partial^{2} u}{\partial x^{2}}=F(u)-T(u, v) \frac{\partial u}{\partial x} \\
\frac{\partial v}{\partial t}-d_{2} \frac{\partial^{2} v}{\partial x^{2}}=H(u, v)
\end{gathered}
$$

represents a model for a population with attractant and has growth-diffusion-chemotaxis type. Some versions of this model were investigated in $[18,19,23]$. For other models, we refer the reader, for instance, to $[1,5,7-9,13,20,24]$.

The purpose of this paper is to study the following quasilinear reaction-diffusion parabolic system:

$$
\begin{gathered}
\frac{\partial u}{\partial t}-d_{1} \Delta u=f_{1}(x, t, u, v, \nabla u, \nabla v)+F(x) \quad \text { in } Q_{T}, \\
\frac{\partial v}{\partial t}-d_{2} \Delta v=f_{2}(x, t, u, v, \nabla u, \nabla v)+G(x) \quad \text { in } Q_{T}, \\
u(x, 0)=u_{0}, \quad v(x, 0)=v_{0} \quad \text { in } \Omega, \\
u=v=0 \quad \text { on } \Sigma_{T},
\end{gathered}
$$

where $d_{1}, d_{2}$ are positive constants, $\Omega$ is an open bounded subset of $\mathbb{R}^{N}$, with smooth boundary $\partial \Omega, Q_{T}=\Omega \times I, T>0$, and $\Sigma_{T}=\partial \Omega \times I, T>0 ; f_{1}, f_{2}: \Omega \times I \times \mathbb{R}^{2} \times \mathbb{R}^{2 N} \rightarrow \mathbb{R}$ are measurable functions.

For the semilinear case of (1.5)-(1.7) (when the functions $f_{1}$ and $f_{2}$ do not depend on the gradient), the existence of positive solutions has been established in $[10-12,16]$, under the following assumptions.

Assumption 1.1. The total mass of the components $u, v$ is controlled with time, which is ensured by

$$
\begin{gathered}
\left(f_{1}+f_{2}\right)(x, t, u, v, p, q) \leq L_{1}(u+v+1), \\
\forall(u, v, p, q) \in \mathbb{R}_{+}^{2} \times \mathbb{R}^{2 N} \quad \text { a.e. }(x, t) \in Q_{T}, L_{1} \geq 0 .
\end{gathered}
$$

Assumption 1.2. The function $f_{1}$ verifies

$$
\begin{gathered}
f_{1}(x, t, u, v, s, r) \leq L_{2}(u+v+1), \\
\forall(u, v, p, q) \in \mathbb{R}_{+}^{2} \times \mathbb{R}^{2 N} \quad \text { a.e. }(x, t) \in Q_{T}, L_{2} \geq 0 .
\end{gathered}
$$


Note that if Assumption 1.1 or 1.2 does not hold, the authors in [21] have been proved blowup in finite time of the solutions to some semilinear reaction-diffusion systems.

As for the quasilinear case, it is showed in [4] the existence of positive weak solutions when the initial data are in $L^{1}$ under Assumptions 1.1 and 1.2.

Assumption 1.3. The positivity of the solution is preserved with time, which is ensured by

$$
\begin{gathered}
f_{1}(x, t, 0, v, p, q), f_{2}(x, t, u, 0, p, q) \geq 0, \\
\forall(u, v, p, q) \in \mathbb{R}_{+}^{2} \times \mathbb{R}^{2 N} \quad \text { a.e. }(x, t) \in Q_{T} .
\end{gathered}
$$

Assumption 1.4. The nonlinear term with respect to the gradient is subquadratic, namely,

$$
\begin{aligned}
& \left|f_{1}(x, t, u, v, \nabla u, \nabla v)\right|+\left|f_{2}(x, t, u, v, \nabla u, \nabla v)\right| \\
& \leq C(|u|,|v|)\left(\|\nabla u\|^{m}+\left\|\nabla z_{1}\right\|^{m}+1\right),
\end{aligned}
$$

where $1 \leq m<2, C:[0, \infty)^{2} \rightarrow[0, \infty)$ is nondecreasing.

A more general result has been obtained later when the initial data are in $L^{2}$ (see [2]). The authors in [2] have investigated problems (1.5)-(1.7) under Assumptions 1.1, 1.3 together with the following assumptions.

Assumption 1.5. The functions $f_{1}$ and $f_{2}$ have critical growth with respect to $|\nabla u|,(m=2)$. Assumption 1.6. The function $f_{1}$ satisfies the "sign condition"

$$
u f_{2}(x, t, u, v, \nabla u, \nabla v) \leq 0 \quad \forall u, v \geq 0 \text {, a.e. }(x, t) \in Q_{T} .
$$

Note that for a single equation $\left(d_{1}=d_{2}\right.$ and $\left.f_{1}=f_{2}\right)$, existence results have been obtained by many authors; see for instance $[1,3,6,15]$. Finally, we mention that in order to establish the existence, many authors have used some regularizations in time and some truncation based on the so-called natural truncation $T_{k}$ defined by

$$
T_{k}(s)=\max (-k, \min (k, s))
$$

where $k$ is a positive real number.

The present paper can be considered as a continuation of works cited above, especially $[2,4]$. Our main goal is to extend those results, in a certain sense. Namely, we will establish the existence, uniqueness, and continuous dependence of a weak solution of problems (1.5)-(1.7) without supposing Assumptions 1.1-1.6. We will consider only the following. Assumption 1.7. The functions $f_{i}(i=1,2)$ are bounded in $L^{2}$ and satisfy

$$
\left|f_{i}\left(\cdot, \cdot, p_{1}, q_{1}\right)-f_{i}\left(\cdot, \cdot, p_{2}, q_{2}\right)\right| \leq L\left(\left|p_{1}-p_{2}\right|+|| q_{1}-q_{2}||\right),
$$

where $L$ is a positive constant. 


\section{Solvability of a class of reaction-diffusion systems}

The paper is organized as follows. In Section 2 we transform problems (1.5)-(1.7) to an equivalent one which is easier to analyze, and we make precise in which sense we solve the reduced problem. Then, in Section 3, we formulate an approximate problem. In Section 4, we derive some useful a priori estimates. Section 5 is devoted to establish the existence of a weak solution, while the uniqueness and continuous dependence of the solution are given in Section 6.

\section{An equivalent problem}

In this section, we will consider the linearization of (1.5)-(1.7) obtained by assuming that $f_{1}=-f_{2} ; d_{1}=d_{2}=d$. The sum of the two components $u$ and $v$ satisfies the following linear parabolic equation:

$$
\begin{gathered}
\frac{\partial w}{\partial t}-d \Delta w=0 \quad \text { in } Q_{T}, \\
w(x, 0)=u_{0}(x)+v_{0}(x) \quad \text { in } \Omega, \\
w=0 \quad \text { on } \Sigma_{T} .
\end{gathered}
$$

Consequently, the function $u$ of problems (1.5)-(1.7) fulfills

$$
\begin{gathered}
\frac{\partial u}{\partial t}-d \Delta u=f_{1}(x, t, u, w-u, \nabla u, \nabla w-\nabla u) \text { in } Q_{T}, \\
u(x, 0)=u_{0}(x) \quad \text { in } \Omega, \\
u=0 \quad \text { on } \Sigma_{T} .
\end{gathered}
$$

It is well known that (2.1) has a unique solution in $L^{2}\left(I ; H_{0}^{1}(\Omega)\right) \cap C\left(I ; L^{2}(\Omega)\right)$ that satisfies $\partial w / \partial t \in L^{2}\left(I ; L^{2}(\Omega)\right)$. Then, if we show that $u$, solution of problem (2.2), exists and sets

$$
v:=w-u
$$

then problems (1.5)-(1.7) will be solved.

Consider now the following auxiliary problem:

$$
\begin{gathered}
\frac{\partial \sigma}{\partial t}-d \Delta \sigma=0 \quad \text { in } Q_{T} \\
\sigma(x, 0)=u_{0}(x) \quad \text { in } \Omega, \\
\sigma=0 \quad \text { on } \Sigma_{T} .
\end{gathered}
$$

Let $z=u-\sigma$, where $u$ is the solution of problem (2.2). Therefore $z$ verifies

$$
\begin{gathered}
\frac{\partial z}{\partial t}-d \Delta z=f(x, t, z, \nabla z) \text { in } Q_{T}, \\
z(x, 0)=0 \quad \text { in } \Omega, \\
z=0 \quad \text { on } \Sigma_{T},
\end{gathered}
$$


where

$$
f(x, t, z, \nabla z)=f_{1}(x, t, z+\sigma, w-z-\sigma, \nabla z+\nabla \sigma, \nabla w-\nabla z-\nabla \sigma) .
$$

Since problem (2.4) possesses a unique solution, our objective is to solve problem (2.5).

Let us now define the notion of the solution we are looking for.

Definition 2.1. Say that $z(x, t)$ is a weak solution of problem (2.5) if the following properties are verified:

(i) $z \in L^{2}\left(I ; H_{0}^{1}(\Omega)\right) \cap C\left(I ; L^{2}(\Omega)\right)$;

(ii) $\partial z / \partial t \in L^{2}\left(I ; L^{2}(\Omega)\right)$;

(iii) $z$ satisfies the initial condition $z(\cdot, 0)=0$ in $L^{2}(\Omega)$;

(iv) the integral identity

$$
\left(\frac{\partial z(\cdot, t)}{\partial t}, \theta(\cdot, t)\right)+d(\nabla z(\cdot, t), \nabla \theta(\cdot, t))=(f(\cdot, t), \theta(\cdot, t))
$$

holds for all $\theta \in H_{0}^{1}(\Omega)$, and all $t \in I$.

As functions $f_{i}(i=1,2)$, the function $f$ verifies the following.

Assumption 2.2. The function $f$ is bounded in $L^{2}$ and satisfies the Lipschitz condition: $\exists L>0$ such

$$
\left|f\left(\cdot, \cdot, p_{1}, q_{1}\right)-f\left(\cdot, \cdot, p_{2}, q_{2}\right)\right| \leq L\left(\left|p_{1}-p_{2}\right|+|| q_{1}-q_{2}||\right) .
$$

\section{Formulation of an approximate problem}

Let $\left\{z_{n}\right\}_{n}$ be a sequence constructed as follows.

For $n=0$, we set $z_{0}(x, t)=0$ for all $(x, t) \in Q_{T}$, the other terms of the sequence are obtained iteratively as solutions of the linear parabolic equation

$$
\begin{gathered}
\frac{\partial z_{n}}{\partial t}-d \Delta z_{n}=f_{n}(x, t) \quad \text { in } Q_{T} \\
z_{n}(x, 0)=0 \quad \text { in } \Omega \\
z_{n}=0 \quad \text { on } \Sigma_{T},
\end{gathered}
$$

where

$$
f_{n}(x, t)=f\left(x, t, z_{n-1}, \nabla z_{n-1}\right)
$$

It is well known that for any fixed " $n$," problem (3.1) has a unique solution $z_{n}$ in $L^{2}(I$; $\left.H_{0}^{1}(\Omega)\right) \cap C\left(I ; L^{2}(\Omega)\right)$, verifying $\partial z_{n} / \partial t \in L^{2}\left(I ; L^{2}(\Omega)\right)$. 
6 Solvability of a class of reaction-diffusion systems

Set

$$
y_{n}(x, t)=z_{n+1}(x, t)-z_{n}(x, t),
$$

one can easily check that $y_{n}$ verifies

$$
\begin{gathered}
\frac{\partial y_{n}}{\partial t}-d \Delta y_{n}=F_{n}(x, t) \quad \text { in } Q_{T}, \\
y_{n}(x, 0)=0 \quad \text { in } \Omega, \\
y_{n}=0 \quad \text { on } \Sigma_{T},
\end{gathered}
$$

where

$$
F_{n}(x, t)=f_{n+1}(x, t)-f_{n}(x, t)
$$

\section{A priori estimates}

In this section, we will establish useful estimates on $y_{n}$ in some suitable spaces in order to prove the convergence of the sequence $\left\{z_{n}\right\}_{n}$ to the solution of problem (3.1). To this end, we consider the weak formulation of problem (3.4),

$$
\left(\frac{\partial y_{n}(\cdot, t)}{\partial t}, \theta(\cdot, t)\right)+d\left(\nabla y_{n}(\cdot, t), \nabla \theta(\cdot, t)\right)=\left(F_{n}(\cdot, t), \theta(\cdot, t)\right)
$$

in which we set $\theta=y_{n}$ and integrate over $(0, \tau)$ to obtain

$$
\left\|y_{n}(\cdot, \tau)\right\|^{2}+2 d \int_{0}^{\tau}\left\|\nabla y_{n}(\cdot, t)\right\|^{2} d t=2 \int_{0}^{\tau}\left(F_{n}(\cdot, t), y_{n}(\cdot, t)\right) d t
$$

According to the Cauchy inequality, it follows

$$
2 d \int_{0}^{\tau}\left\|\nabla y_{n}(\cdot, t)\right\|^{2} d t+\left\|y_{n}(\cdot, \tau)\right\|^{2}=\int_{0}^{\tau}\left\|F_{n}(\cdot, t)\right\|^{2} d t+\int_{0}^{\tau}\left\|y_{n}(\cdot, t)\right\|^{2} d t .
$$

The application of a lemma of Gronwall's type leads to

$$
2 d \int_{0}^{\tau}\left\|\nabla y_{n}(\cdot, t)\right\|^{2} d t+\left\|y_{n}(\cdot, \tau)\right\|^{2} \leq e^{T} \int_{0}^{T}\left\|F_{n}(\cdot, t)\right\|_{H_{0}^{1}(\Omega)}^{2} d t .
$$

Therefore, by omitting the second term on the left-hand side of (4.4) and applying Assumption 2.2 to the right-hand side, we get

$$
d \int_{0}^{\tau}\left\|\nabla y_{n}(\cdot, t)\right\|^{2} d t \leq 2 e^{T} L^{2} \int_{0}^{T}\left\|y_{n-1}(\cdot, t)\right\|_{H_{0}^{1}(\Omega)}^{2} d t .
$$

In light of the Friedrichs inequality [22], we have

$$
\int_{0}^{\tau}\left\|y_{n}(\cdot, t)\right\|_{H_{0}^{1}(\Omega)}^{2} d t \leq \frac{2 e^{T} L^{2} C_{\Omega}}{d} \int_{0}^{T}\left\|y_{n-1}(\cdot, t)\right\|_{H_{0}^{1}(\Omega)}^{2} d t .
$$


It follows that

$$
\left\|y_{n}\right\|_{L^{2}\left(I, H_{0}^{1}(\Omega)\right)} \leq L c_{1}\left\|y_{n-1}\right\|_{L^{2}\left(I, H_{0}^{1}(\Omega)\right)},
$$

where

$$
c_{1}=\frac{2^{1 / 2} e^{T / 2} C_{\Omega}^{1 / 2}}{d^{1 / 2}} .
$$

On the other hand, by virtue of (4.1) in which we set $\theta=\partial y_{n} / \partial t$, it yields

$$
2 \int_{0}^{\tau}\left\|\frac{\partial y_{n}(\cdot, t)}{\partial t}\right\|^{2} d t+d\left\|\nabla y_{n}(\cdot, t)\right\|^{2} d t=2 \int_{0}^{\tau}\left(F_{n}(\cdot, t), \frac{\partial y_{n}(\cdot, t)}{\partial t}\right) d t
$$

In light of the Cauchy inequality and Assumption 2.2, the right-hand side of (4.9) is then dominated by

$$
\int_{0}^{\tau}\left\|\frac{\partial y_{n}(\cdot, t)}{\partial t}\right\|^{2} d t+2 L^{2} \int_{0}^{\tau}\left\|y_{n-1}(\cdot, t)\right\|_{H_{0}^{1}(\Omega)}^{2} d t
$$

where the integral over $\partial y_{n} / \partial t$ will be absorbed in the left-hand side of (4.9). Thanks to the Friedrichs' inequality the second term on the left-hand side of (4.9) is controlled from below by

$$
d C_{\Omega}^{-1}\left\|y_{n}(\cdot, \tau)\right\|_{H_{0}^{1}(\Omega)}^{2}
$$

Therefore, we have

$$
\begin{aligned}
& \int_{0}^{\tau}\left\|\frac{\partial y_{n}(\cdot, t)}{\partial t}\right\|^{2} d t+d C_{\Omega}^{-1}\left\|y_{n}(\cdot, \tau)\right\|_{H_{0}^{1}(\Omega)}^{2} \\
& \quad \leq 2 L^{2} \int_{0}^{T}\left\|y_{n-1}(\cdot, t)\right\|_{H_{0}^{1}(\Omega)}^{2} d t \\
& \quad \leq 2 T L^{2}\left\|y_{n-1}\right\|_{C\left(I ; H_{0}^{1}(\Omega)\right)}^{2} \\
& \quad \leq 2 T L^{2}\left(\left\|y_{n-1}\right\|_{C\left(I ; H_{0}^{1}(\Omega)\right)}^{2}+\left\|\frac{\partial y_{n-1}}{\partial t}\right\|_{L^{2}\left(I ; L^{2}(\Omega)\right)}^{2}\right) .
\end{aligned}
$$

The right-hand side here is independent of $\tau$, hence replacing the left-hand side by its upper bound with respect to $\tau$ from 0 to $T$, thus we obtain

$$
\begin{aligned}
& \left\|\frac{\partial y_{n}}{\partial t}\right\|_{L^{2}\left(I ; L^{2}(\Omega)\right)}^{2}+\left\|y_{n}\right\|_{C\left(I ; H_{0}^{1}(\Omega)\right)}^{2} \\
& \quad \leq \frac{2 L^{2} T}{\min \left(1, d C_{\Omega}^{-1}\right)}\left(\left\|\frac{\partial y_{n-1}}{\partial t}\right\|_{L^{2}\left(I ; L^{2}(\Omega)\right)}^{2}+\left\|y_{n-1}\right\|_{C\left(I ; H_{0}^{1}(\Omega)\right)}^{2}\right),
\end{aligned}
$$

implying finally

$$
\left\|y_{n}\right\|_{B} \leq L c_{2}\left\|y_{n-1}\right\|_{B}
$$


8 Solvability of a class of reaction-diffusion systems

where

$$
c_{2}=\sqrt{\frac{2 T}{\min \left(1, d C_{\Omega}^{-1}\right)}} .
$$

Hence we can present the following theorem.

Theorem 4.1. Suppose that Assumption 2.2 is fulfilled. Then the following estimates hold, for $n=1,2, \ldots$ :

$$
\left\|y_{n}\right\|_{L^{2}\left(I, H_{0}^{1}(\Omega)\right)} \leq L c_{1}\left\|y_{n-1}\right\|_{L^{2}\left(I, H_{0}^{1}(\Omega)\right)}, \quad\left\|y_{n}\right\|_{B} \leq L c_{2}\left\|y_{n-1}\right\|_{B}
$$

where $B$ is the Banach space endowed with the finite norm

$$
\|y\|_{B}=\left(\left\|\frac{\partial y}{\partial t}\right\|_{L^{2}\left(I ; L^{2}(\Omega)\right)}^{2}+\|y\|_{C\left(I ; H_{0}^{1}(\Omega)\right)}^{2}\right)^{1 / 2},
$$

$c_{1}$ and $c_{2}$ are positive constants defined by (4.8) and (4.15).

\section{Convergence and existence result}

TheOREm 5.1. Take the assumption of Theorem 4.1. If

$$
L c_{3}<1
$$

then there exists a pair

$$
\left(z, \frac{\partial z}{\partial t}\right) \in L^{2}\left(I, H_{0}^{1}(\Omega)\right) \cap C\left(I ; L^{2}(\Omega)\right) \times L^{2}\left(I ; L^{2}(\Omega)\right),
$$

verifying

$$
\left(z_{n}, \frac{\partial z_{n}}{\partial t}\right) \underset{n \rightarrow \infty}{\longrightarrow}\left(z, \frac{\partial z}{\partial t}\right)
$$

where $c_{3}=\max \left(c_{1}, c_{2}\right)$.

Proof. In inequality (4.7), if $L c_{1}<1$, then the series $\sum_{n=0}^{\infty} y_{n}$ converges in $L^{2}\left(I, H_{0}^{1}(\Omega)\right)$. Observe that

$$
z_{n}=S_{n}=\sum_{k=0}^{n-1}\left(z_{k+1}-z_{k}\right),
$$

hence by passing to the limit, we have

$$
z_{n} \longrightarrow \tilde{z} \quad \text { in } L^{2}\left(I, H_{0}^{1}(\Omega)\right)
$$

Since $H_{0}^{1}(\Omega) \hookrightarrow L^{2}(\Omega)$, we have also

$$
z_{n} \longrightarrow \tilde{z} \quad \text { in } L^{2}\left(I, L^{2}(\Omega)\right) .
$$


On the other hand, in estimate (4.14), if $L c_{2}<1$, we deduce that the series $\sum_{n=0}^{\infty} y_{n}$ and thus the sequence $\left\{z_{n}\right\}_{n}$ converge in the space $B$. According to the definition of space $B$ (see Theorem 4.1), we deduce that

$$
\frac{\partial z_{n}}{\partial t} \underset{n \rightarrow \infty}{\longrightarrow} \varphi \quad \text { in } L^{2}\left(I ; L^{2}(\Omega)\right)
$$

We have to prove that $\varphi$ equals $\partial \tilde{z} / \partial t$ in $L^{2}\left(I ; L^{2}(\Omega)\right)$. To this end, we consider the identity

$$
z_{n}(\cdot, t)=\int_{0}^{t} \frac{\partial z_{n}}{\partial \tau} d \tau \quad \forall t \in I
$$

Then, by passing to the limit in (5.8), when $n$ tends to infinity by taking (5.6) and (5.7) into account, it yields

$$
\widetilde{z}(\cdot, t)=\int_{0}^{t} \varphi d \tau \quad \forall t \in I
$$

from which we conclude, see for instance [14, Lemmas 1.3.2 and 1.3.6], that

$$
\tilde{z} \in C\left(I ; L^{2}(\Omega)\right)
$$

differentiable for almost everywhere $t \in I$ and $\partial \tilde{z} / \partial t=\varphi$ in $L^{2}\left(I ; L^{2}(\Omega)\right)$, namely,

$$
\frac{\partial z_{n}}{\partial t} \underset{n \rightarrow \infty}{\longrightarrow} \frac{\partial \tilde{z}}{\partial t} \text { in } L^{2}\left(I ; L^{2}(\Omega)\right) .
$$

Consequently, for $L c_{3}<1$ the limit relation (5.3) is satisfied.

Theorem 5.2. Suppose that assumption of Theorem 4.1 is satisfied, moreover assume that $f(x, t, 0,0) \in L^{2}\left(I ; L^{2}(\Omega)\right)$. Then the limit function $\tilde{z}=\widetilde{z}(x, t)$ is the weak solution of problem (2.5) in the sense of Definition 2.1.

Proof. According to Theorem 5.1, assertions (i) and (ii) of Definition 2.1 are fulfilled. Moreover, from (5.9) we conclude that $\widetilde{z}(\cdot, 0)=0$ holds in $L^{2}(\Omega)$, and so assertion (iii) is verified. It remains to prove that $\tilde{z}$ satisfies the integral identity (iv). Since $z_{n}$ is the solution of (3.1), we have for all $\theta \in L^{2}\left(I ; H_{0}^{1}(\Omega)\right)$,

$$
\left(\frac{\partial z_{n}}{\partial t}, \theta\right)_{L^{2}\left(I ; L^{2}(\Omega)\right)}+d\left(\nabla z_{n}, \nabla \theta\right)_{L^{2}\left(I ; L^{2}(\Omega)\right)}=\left(f_{n}, \theta\right)_{L^{2}\left(I ; L^{2}(\Omega)\right)}
$$

which can be written

$$
\begin{aligned}
\left(\frac{\partial z_{n}}{\partial t}-\frac{\partial \tilde{z}}{\partial t}, \theta\right)_{L^{2}\left(I ; L^{2}(\Omega)\right)} & +\left(\frac{\partial \tilde{z}}{\partial t}, \theta\right)_{L^{2}\left(I ; L^{2}(\Omega)\right)}+d\left(\nabla z_{n}-\nabla \tilde{z}, \nabla \theta\right)_{L^{2}\left(I ; L^{2}(\Omega)\right)} \\
& +d(\nabla \tilde{z}, \nabla \theta)_{L^{2}\left(I ; L^{2}(\Omega)\right)}=\left(f_{n}-f, \theta\right)_{L^{2}\left(I ; L^{2}(\Omega)\right)}+(f, \theta)_{L^{2}\left(I ; L^{2}(\Omega)\right)}
\end{aligned}
$$


10 Solvability of a class of reaction-diffusion systems

If we show that

$$
\begin{gathered}
I_{1}=\left(\frac{\partial z_{n}}{\partial t}-\frac{\partial \tilde{z}}{\partial t}, \theta\right)_{L^{2}\left(I ; L^{2}(\Omega)\right)}+d\left(\nabla z_{n}-\nabla \tilde{z}, \nabla \theta\right)_{L^{2}\left(I ; L^{2}(\Omega)\right)} \underset{n \rightarrow \infty}{\longrightarrow} 0 \\
I_{2}=\left(f_{n}-f, \theta\right)_{L^{2}\left(I ; L^{2}(\Omega)\right)} \underset{n \rightarrow \infty}{\longrightarrow} 0
\end{gathered}
$$

$\tilde{z}$ will be a solution of (2.5) in the sense of Definition 2.1.

For $I_{1}$, we have

$$
\begin{aligned}
I_{1}= & \left(\frac{\partial z_{n}}{\partial t}-\frac{\partial \tilde{z}}{\partial t}, \nabla \theta\right)_{L^{2}\left(I ; L^{2}(\Omega)\right)}+\left(\nabla z_{n}-\nabla \tilde{z}, \nabla \theta\right)_{L^{2}\left(I ; L^{2}(\Omega)\right)} \\
\leq & \left\|\frac{\partial z_{n}}{\partial t}-\frac{\partial \tilde{z}}{\partial t}\right\|\left\|_{L^{2}\left(I ; L^{2}(\Omega)\right)}\right\| \theta \|_{L^{2}\left(I ; L^{2}(\Omega)\right)} \\
& +\left\|\nabla z_{n}-\nabla \tilde{z}\right\|_{L^{2}\left(I ; L^{2}(\Omega)\right)}\|\nabla \theta\|_{L^{2}\left(I ; L^{2}(\Omega)\right)} \\
\leq & \left(\left\|\frac{\partial z_{n}}{\partial t}-\frac{\partial \tilde{z}}{\partial t}\right\|_{L^{2}\left(I ; L^{2}(\Omega)\right)}+\left\|z_{n}-\tilde{z}\right\|_{L^{2}\left(I ; H_{0}^{1}(\Omega)\right)}\right)\|\theta\|_{L^{2}\left(I ; H_{0}^{1}(\Omega)\right)} .
\end{aligned}
$$

Thanks to (5.3) we obtain $\lim _{n \rightarrow \infty} I_{1}=0$. For the remaining term $I_{2}$, we use the Schwarz inequality and Assumption 2.2 to get

$$
\begin{aligned}
I_{2} & =\left(f_{n}-f, \theta\right)_{L^{2}\left(I ; L^{2}(\Omega)\right)} \\
& \leq\left\|f_{n}-f\right\|_{L^{2}\left(I ; L^{2}(\Omega)\right)}\|\theta\|_{L^{2}\left(I ; L^{2}(\Omega)\right)} \\
& =\left\|f_{n}\left(\cdot, \cdot, z_{n-1}, \nabla z_{n-1}\right)-f(\cdot, \cdot, \tilde{z}, \nabla \tilde{z})\right\|_{L^{2}\left(I ; L^{2}(\Omega)\right)}\|\theta\|_{L^{2}\left(I ; L^{2}(\Omega)\right)} \\
& \leq L\left[\left\|z_{n-1}-\tilde{z}\right\|_{L^{2}\left(I ; L^{2}(\Omega)\right)}+\left\|\nabla z_{n-1}-\nabla \tilde{z}\right\|_{L^{2}\left(I ; L^{2}(\Omega)\right)}\right]\|\theta\|_{L^{2}\left(I ; L^{2}(\Omega)\right)} \\
& \leq L\left\|z_{n-1}-\tilde{z}\right\|_{L^{2}\left(I ; H_{0}^{1}(\Omega)\right)}\|\theta\|_{L^{2}\left(I ; L^{2}(\Omega)\right)} .
\end{aligned}
$$

Therefore by passing to the limit, we obtain $\lim _{n \rightarrow \infty} I_{2}=0$. This completes the proof of Theorem 5.2.

\section{Uniqueness and continuous dependence}

Theorem 6.1. Suppose that assumptions of Theorem 5.2 are fulfilled. Let $z_{1}$ and $z_{2}$ be two weak solutions of $(2.5)$ in $L^{2}\left(I ; H_{0}^{1}(\Omega)\right)$. Then

$$
\left\|z_{1}-z_{2}\right\|_{L^{2}\left(I ; H_{0}^{1}(\Omega)\right)} \leq L c_{1}\left\|z_{1}-z_{2}\right\|_{L^{2}\left(I ; H_{0}^{1}(\Omega)\right)}
$$

where $c_{1}$ is defined by (4.8). 
Proof. Let $y=z_{1}-z_{2}$, it is clear that $y$ satisfies $t \in I$,

$$
\begin{gathered}
\frac{\partial y}{\partial t}-d \Delta y=f\left(x, t, z_{1}, \nabla z_{1}\right)-f\left(x, t, z_{2}, \nabla z_{2}\right) \text { in } Q_{T} \\
y(x, 0)=0 \quad \text { in } \Omega, \\
y=0 \text { on } \Sigma_{T} .
\end{gathered}
$$

Considering the weak formulation of problem (6.2) and performing a similar calculation to that for the establishment of estimate (4.7), we derive the desired result.

As a consequence of Theorem 6.1, we obtain the following.

COROLlary 6.2. Under assumptions of Theorem 6.1, the solution of problem (2.5) is unique. Proof. The proof is obvious, it suffices to observe that by Theorem 6.1,

$$
\left(1-L c_{1}\right)\|y\|_{L^{2}\left(I ; H_{0}^{1}(\Omega)\right)} \leq 0,
$$

and by assumption (5.1) $1-L c_{1}>0$. Consequently, $y=0$, which means $z_{1}=z_{2}$, which achieves the proof.

Theorem 6.3. Suppose that $u=u(x, t)$ and $u^{*}=u^{*}(x, t)$ are two solutions corresponding to $\left(u_{0}, f\right)$ and $\left(u_{0}^{*}, f^{*}\right)$. Moreover, assume that

$$
\left|f_{1}\left(\cdot, t, p_{1}, q_{1}\right)-f_{1}^{*}\left(\cdot, t, p_{2}, q_{2}\right)\right| \leq K(t)+L\left(\left\|p_{1}-p_{2}\right\|+\| q_{1}-q_{2}||\right),
$$

for some continuous nonnegative function $K(t)$ and certain constant $L$. Then

$$
\left\|u(\cdot, t)-u^{*}(\cdot, t)\right\|^{2} \leq\left(\left\|u_{0}-u_{0}^{*}\right\|^{2}+\int_{0}^{t} K^{2}(\tau) d \tau\right) e^{c_{4} t}
$$

for all $t \in I$, where

$$
c_{4}=\max \left(\frac{2}{L^{2}}, 1\right) \exp \left(\left(\frac{3 L^{2}}{2}+2\right) \tau\right)
$$

Proof. Considering the weak formulation of problem (2.2) written for $u$, subtracting from it the same integral identity written for $u^{*}$ and putting $\theta=u-u^{*}$, and performing an integration by parts, we get

$$
\begin{aligned}
& \frac{\partial}{\partial t}\left\|u(\cdot, t)-u^{*}(\cdot, t)\right\|^{2}+2\left\|\nabla u(\cdot, t)-\nabla u^{*}(\cdot, t)\right\|^{2} \\
& \quad=2\left(f_{1}(\cdot, t, u(\cdot, t), \nabla u(\cdot, t))-f_{1}^{*}\left(\cdot, t, u^{*}(\cdot, t), \nabla u^{*}(\cdot, t)\right), u(\cdot, t)-u^{*}(\cdot, t)\right) .
\end{aligned}
$$


12 Solvability of a class of reaction-diffusion systems

Integrating (6.7) over $(0, t)$, applying the Cauchy inequality, and using assumption (6.4), we obtain

$$
\begin{aligned}
\| u(\cdot, t) & -u^{*}(\cdot, t)\left\|^{2}+2 \int_{0}^{t}\right\| \nabla u(\cdot, \tau)-\nabla u^{*}(\cdot, \tau) \|^{2} \\
\leq & \left\|u_{0}-u_{0}^{*}\right\|^{2}+3 \varepsilon \int_{0}^{t} K^{2}(\tau) d \tau \\
& +\left(\frac{1}{\varepsilon}+3 \varepsilon L^{2}\right) \int_{0}^{t}\left\|u(\cdot, \tau)-u^{*}(\cdot, \tau)\right\|^{2} d \tau \\
& +3 \varepsilon L^{2} \int_{0}^{t}\left\|\nabla u(\cdot, \tau)-\nabla u^{*}(\cdot, \tau)\right\|^{2} d \tau .
\end{aligned}
$$

Choosing $\varepsilon=2 / 3 L^{2}$ and applying Gronwall's lemma to the obtained inequality, we get estimate (6.5), which completes the proof.

\section{Generalizations}

(i) The above results can be extended for more general equations of the type of (1.5), (1.6) with higher-order derivatives and coefficients depending on $x$ and $t$, namely, for the following problem:

$$
\begin{aligned}
& \frac{\partial u}{\partial t}-\sum_{|i|,|j| \leq m}(-1)^{|i|} D^{i}\left(a_{i j}^{(1)}(x) D^{j} u\right) \\
& \quad=f_{1}\left(x, t, u, v, \nabla u, \nabla v, \ldots, \nabla^{m-1} u, \nabla^{m-1} v\right)+F(x) \text { in } Q_{T}, \\
& \frac{\partial v}{\partial t}-\sum_{|i|,|j| \leq m}(-1)^{|i|} D^{i}\left(a_{i j}^{(2)}(x) D^{j} v\right) \\
& \quad=f_{2}\left(x, t, u, v, \nabla u, \nabla v, \ldots, \nabla^{m-1} u, \nabla^{m-1} v\right)+G(x) \text { in } Q_{T},
\end{aligned}
$$

since the compactness $H_{0}^{m}(\Omega) \hookrightarrow H^{m-1}(\Omega)$ can be used, where $a_{i j}^{(k)}(x)(k=1,2)$ are such that $\sum_{|i|,|j| \leq m} a_{i j}^{(k)} \xi_{i} \xi_{j} \geq C \sum_{|i|=m} \xi_{i}^{2}, a_{i j}^{(k)}=a_{j i}^{(k)}$ for all couples $|i| \geq 1,|j| \geq 1, a_{i j}^{(k)}$ a Lipschitz continuous in $t$ on $\bar{Q}(|i|,|j| \leq m)$. Here, homogeneous Dirichlet boundary conditions

$$
\frac{\partial^{s} u}{\partial \vartheta^{s}}=\frac{\partial^{s} v}{\partial \vartheta^{s}}=0 \quad \text { on } \partial \Omega
$$

for $s=0,1, \ldots, m-1$, are considered instead of $\left(1.1_{4}\right)$. 
(ii) Our results make it possible to study the boundary value problem for a quasilinear pluriparabolic system, that is a system with the form

$$
\begin{gathered}
\sum_{i=1}^{n} \frac{\partial u}{\partial t_{i}}-d_{1} \Delta u=f_{1}(x, t, u, v, \nabla u, \nabla v)+F(x) \quad \text { in } Q_{T}, \\
\sum_{i=1}^{n} \frac{\partial v}{\partial t_{i}}-d_{2} \Delta v=f_{2}(x, t, u, v, \nabla u, \nabla v)+G(x) \quad \text { in } Q_{T}, \\
u\left(x, t^{i, 0}\right)=\Phi_{i}\left(x, t^{i}\right), \quad v\left(x, t^{i, 0}\right)=\Psi_{i}\left(x, t^{i}\right) \quad \text { for }(x, t) \in Q_{0}^{i}, \\
u=v=0, \quad \text { on } \Sigma_{T},
\end{gathered}
$$

or more generally for a quasilinear pluriparabolic system with nonlocal initial conditions

$$
\begin{gathered}
\sum_{i=1}^{n} \frac{\partial u}{\partial t_{i}}-d_{1} \operatorname{sing} \prod_{i=1}^{n}\left(1-\left\|\lambda_{i}\right\|^{2}\right) \Delta u=f_{1}(x, t, u, v, \nabla u, \nabla v)+F(x) \quad \text { in } Q_{T} \\
\sum_{i=1}^{n} \frac{\partial v}{\partial t_{i}}-d_{2} \operatorname{sing} \prod_{i=1}^{n}\left(1-\left\|\lambda_{i}\right\|^{2}\right) \Delta v=f_{2}(x, t, u, v, \nabla u, \nabla v)+G(x) \quad \text { in } Q_{T} \\
\left(\ell_{i} u\right)(x, t):=u\left(x, t^{i, 0}\right)-\lambda_{i} u\left(x, t^{i, T}\right)=\Phi_{i}\left(x, t^{i}\right) \quad \text { in } Q_{0}^{i}, \\
\left(\ell_{i} v\right)(x, t):=v\left(x, t^{i, 0}\right)-\lambda_{i} v\left(x, t^{i, T}\right)=\Psi_{i}\left(x, t^{i}\right) \quad \text { in } Q_{0}^{i}, \\
u=v=0, \quad \text { on } \Sigma_{T},
\end{gathered}
$$

where

$$
\begin{gathered}
t=\left(t_{1}, \ldots, t_{n}\right), \quad t^{i}=\left(t_{1}, \ldots, t_{i-1}, t_{i+1}, \ldots, t_{n}\right), \\
t^{i, 0}=\left(t_{1}, \ldots, t_{i-1}, 0, t_{i+1}, \ldots, t_{n}\right), \\
t^{i, T}=\left(t_{1}, \ldots, t_{i-1}, T_{i}, t_{i+1}, \ldots, t_{n}\right), \\
(i=1, \ldots, n) \quad I_{i}=\left(0, T_{i}\right), \quad T_{i}<\infty(i=1, \ldots, n), I=\prod_{i=1}^{n} I_{i}, \\
Q_{0}^{i}=I_{1} \times \cdots \times I_{i-1} \times\{0\} \times I_{i+1} \times \cdots \times I_{n} \quad(i=1, \ldots, n) .
\end{gathered}
$$

\section{Acknowledgment}

This work has been done in "The Abdus Salam International Centre of Theoretical Physics," Trieste, Italy. 


\section{References}

[1] N. Alaa, Solutions faibles d'équations paraboliques quasilinéaires avec données initiales mesures [Weak solutions of quasilinear parabolic equations with measures as initial data], Annales Mathématiques Blaise Pascal 3 (1996), no. 2, 1-15.

[2] N. Alaa and I. Mounir, Global existence for reaction-diffusion systems with mass control and critical growth with respect to the gradient, Journal of Mathematical Analysis and Applications 253 (2001), no. 2, 532-557.

[3] L. Boccardo, F. Murat, and J.-P. Puel, Existence results for some quasilinear parabolic equations, Nonlinear Analysis. Theory, Methods \& Applications. Series A 13 (1989), no. 4, 373-392.

[4] N. Boudiba, Existence globale pour des systèmes de réaction-diffusion paraboliques quasilinéaires, Thèse de troisième cycle, Université des Sciences et de la Technologie Houari Boumediene d'Alger, Algérie, 1995.

[5] N. F. Britton, Reaction-Diffusion Equations and Their Applications to Biology, Academic Press, New York; Harcourt Brace Jovanovich, London, 1986.

[6] A. Dall'Aglio and L. Orsina, Nonlinear parabolic equations with natural growth conditions and $L^{1}$ data, Nonlinear Analysis. Theory, Methods \& Applications. Series A 27 (1996), no. 1, 59-73.

[7] J. Dockery, V. Hutson, K. Mischaikow, and M. Pernarowski, The evolution of slow dispersal rates: a reaction diffusion model, Journal of Mathematical Biology 37 (1998), no. 1, 61-83.

[8] L. Edelstein-Keshet, Mathematical Models in Biology, McGraw-Hill, New York, 1987.

[9] P. C. Fife, Mathematical Aspects of Reacting and Diffusing Systems, Lecture Notes in Biomathematics, vol. 28, Springer, Berlin, 1979.

[10] A. Haraux and A. Youkana, On a result of K. Masuda concerning reaction-diffusion equations, The Tôhoku Mathematical Journal. Second Series 40 (1988), no. 1, 159-163.

[11] S. L. Hollis, R. H. Martin Jr., and M. Pierre, Global existence and boundedness in reaction-diffusion systems, SIAM Journal on Mathematical Analysis 18 (1987), no. 3, 744-761.

[12] S. L. Hollis and J. Morgan, Interior estimates for a class of reaction-diffusion systems from $L^{1}$ a priori estimates, Journal of Differential Equations 98 (1992), no. 2, 260-276.

[13] V. Hutson, J. López-Gómez, K. Mischaikow, and G. Vickers, Limit behaviour for a competing species problem with diffusion, Dynamical Systems and Applications (R. P. Agarwal, ed.), World Sci. Ser. Appl. Anal., vol. 4, World Scientific, New Jersey, 1995, pp. 343-358.

[14] J. Kačur, Method of Rothe in Evolution Equations, Teubner Texts in Mathematics, vol. 80, BSB B. G. Teubner, Leipzig, 1985.

[15] R. Landes and V. Mustonen, On parabolic initial-boundary value problems with critical growth for the gradient, Annales de l'Institut Henri Poincaré. Analyse Non Linéaire 11 (1994), no. 2, $135-158$.

[16] R. H. Martin Jr. and M. Pierre, Nonlinear reaction-diffusion systems, Nonlinear Equations in the Applied Sciences (W. F. Ames and C. Rogers Kapell, eds.), Math. Sci. Engrg., vol. 185, Academic Press, Massachusetts, 1992, pp. 363-398.

[17] M. Mimura and J. D. Murray, On a diffusive prey-predator model which exhibits patchiness, Journal of Theoretical Biology 75 (1978), no. 3, 249-262.

[18] P. B. Monk and H. G. Othmer, Cyclic AMP oscillations in suspensions of Dictyostelium discoideum, Philosophical Transactions of the Royal Society of London. Series B, Biological Sciences 323 (1989), no. 1215, 185-224.

[19] J. D. Murray, Mathematical Biology, 2nd ed., Biomathematics, vol. 19, Springer, Berlin, 1993.

[20] E. S. Oran and J. P. Boris, Numerical Simulation of Reactive Flow, Elsevier, New York, 1987.

[21] M. Pierre and D. Schmitt, Existence globale ou explosion pour les systèmes de réaction-diffusion avec contrôle de masse, Thèse de Doctorat, Université Henri Poincaré, Nancy I, Nancy, 1995.

[22] K. Rektorys, Variational Methods in Mathematics, Science and Engineering, D. Reidel, Dordrecht, 1982.

[23] A. A. Samarskiı̌ and A. P. Mikhălov, Mathematical Modeling, Fizmatlit "Nauka", Moscow, 1997. 
[24] J. Smoller, Shock Waves and Reaction-Diffusion Equations, Fundamental Principles of Mathematical Science, vol. 258, Springer, New York, 1983.

[25] E. H. Twizell, Y. Wang, W. G. Price, and F. Fakhr, Finite-difference methods for solving the reactiondiffusion equations of a simple isothermal chemical system, Numerical Methods for Partial Differential Equations 10 (1994), no. 4, 435-454.

Abdelfatah Bouziani: Department of Mathematics, Larbi Ben M'hidi University Centre, Oum El Bouagui, P.O. Box 565, Oum El Bouagui 04000, Algeria

E-mail address: aefbouziani@yahoo.fr

Ilham Mounir: Department of Mathematics, Faculty of Sciences Semlalia,

Cadi Ayyad University, B.P. 2390, Morocco

E-mail address: ilhamounir@ucam.ac.ma 


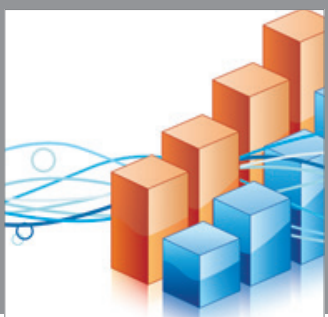

Advances in

Operations Research

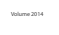

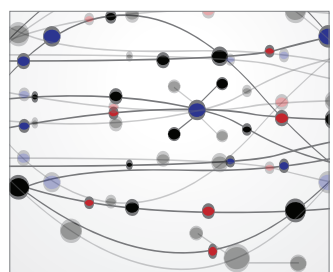

\section{The Scientific} World Journal
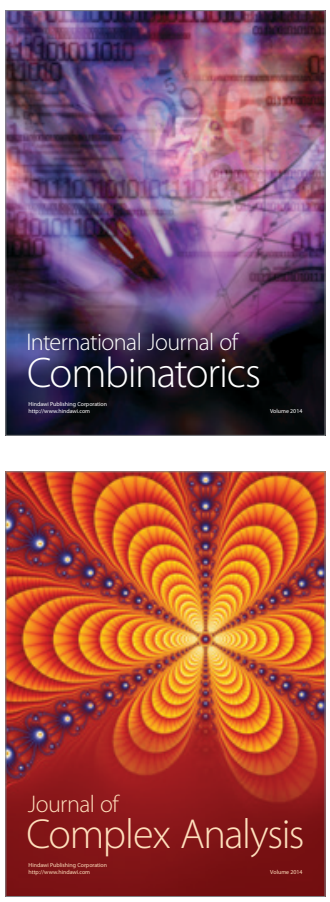

International Journal of

Mathematics and

Mathematical

Sciences
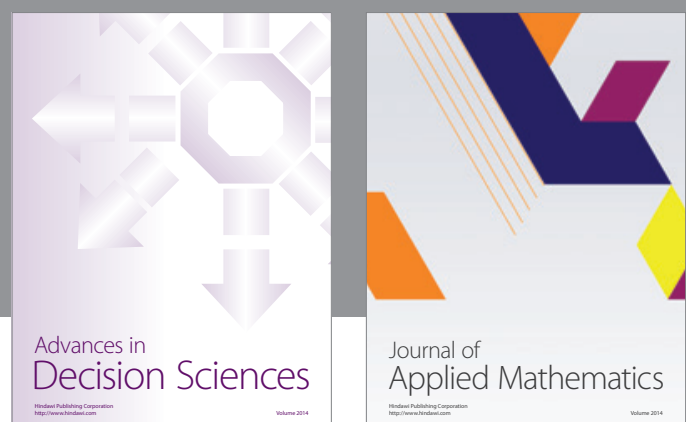

Journal of

Applied Mathematics
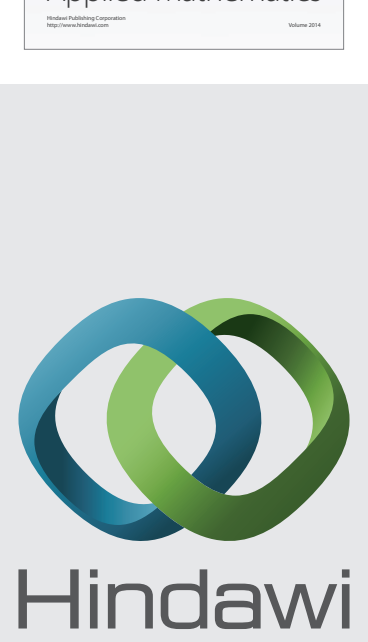

Submit your manuscripts at http://www.hindawi.com
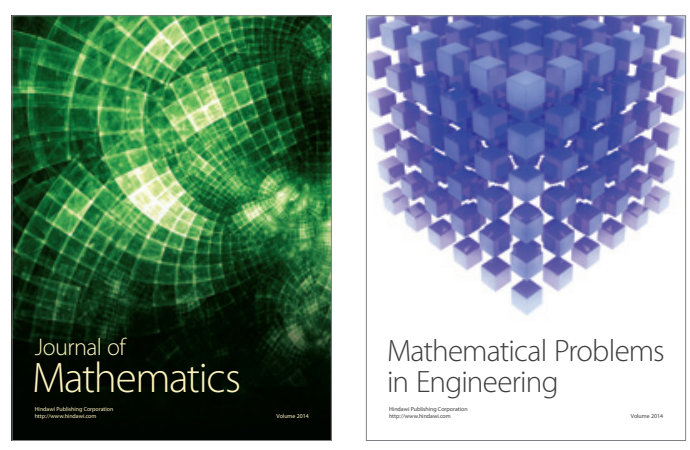

Mathematical Problems in Engineering
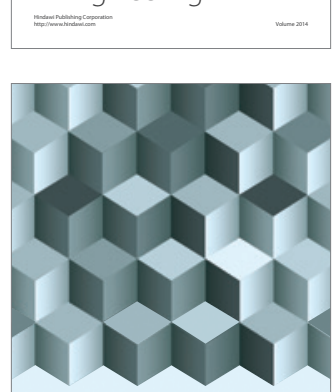

Journal of

Function Spaces
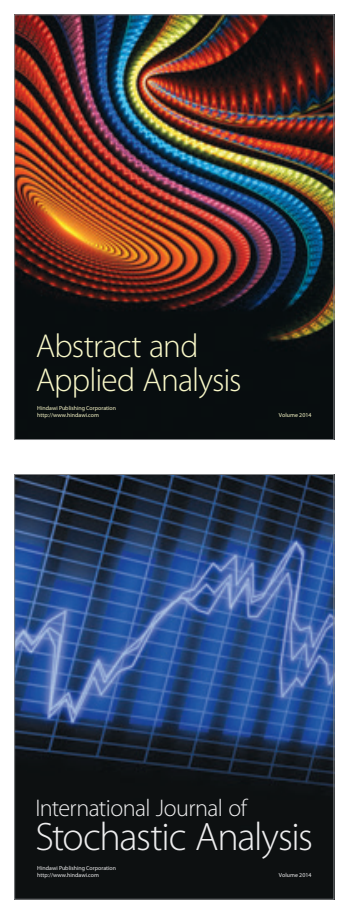

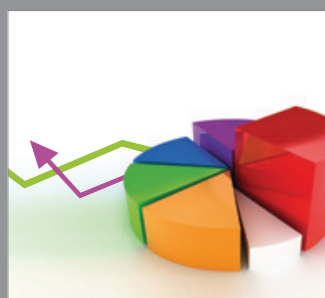

ournal of

Probability and Statistics

Promensencen
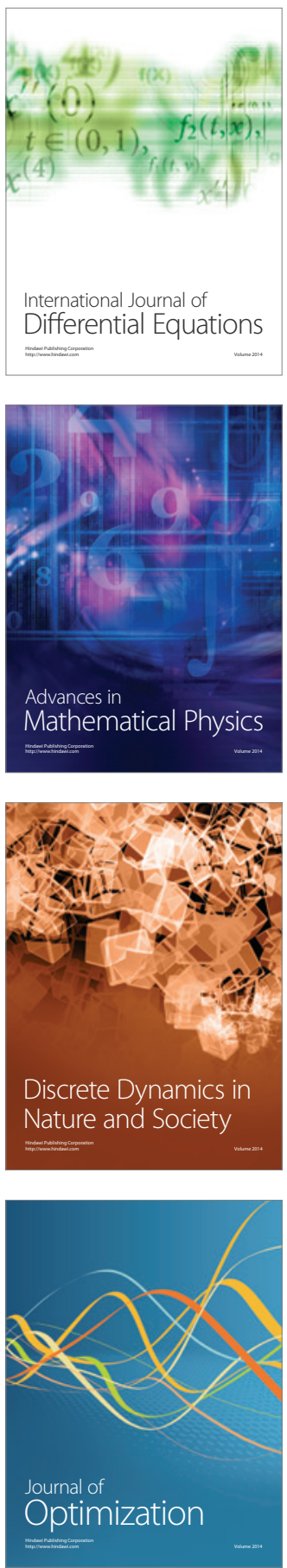